\title{
VIII. CARBONATE CONTENT, CARBONATE MINERALOGY, AND BULK MINERALOGY OF DSDP LEG 42A SAMPLES
}

\author{
Jens Müller, Lehrstuhl für Geologie, Technische Universität, München, West Germany
}

For the determination of total carbonate and for $\mathrm{x}$-ray diffraction analysis samples were dried $\left(40^{\circ} \mathrm{C}\right)$ and ground to a size of less than $40 \mu \mathrm{m}$.

Inorganic carbon was analyzed volumetrically with a LECO-analyzer. Accuracy of this method is $\pm 1 \%$ (See Sigl, this volume).

$\mathrm{X}$-ray analysis was made with unoriented powder specimens with a scanning speed of $1^{\circ} 2 \theta / \mathrm{min}$; when Mg-calcite ( $>4$ mol- $\% \mathrm{MgCO}_{3}$ ) was present, scanning speed was reduced to $1 / 2^{\circ} 2 \theta / \mathrm{min}$ to obtain better resolution. The ratios of aragonite, calcite, and $\mathrm{Mg}$ calcite were determined by measuring peak areas of the different carbonate minerals and calculated using calibration curves published elsewhere (Müller and Müller, 1967; Müller, 1969). The calcite-dolomite ratio was determined after the method described by Tennant and Berger (1957). The proportions of carbonate minerals were calculated using the inorganic carbon determined by the LECO-method and by the ratios obtained by X-ray diffraction. The precision of this method is $\pm 5 \%$ by weight.

The $\mathrm{MgCO}_{3}$-content of the $\mathrm{Mg}$-calcite and the composition of the dolomite were determined by the shift of the (104) peak of calcite and of dolomite, respectively (Goldsmith and Graf, 1958; Füchtbauer and Goldschmidt, 1965) using the position of the (101) peak of quartz as an internal standard. Many samples contain both stoichiometric dolomite $\left(\mathrm{Ca}_{50} \mathrm{Mg}_{50}\right)$ and Ca-dolomite (usually $\mathrm{Ca}_{54} \mathrm{Mg}_{46}$ ), the latter being more frequent and abundant.

The proportions of quartz, K-feldspar, plagioclase, gypsum, anhydrite, and pyrite were estimated by their peak heights assigning arbitrary symbols for their abundance. The respective symbols cover the following ranges $\left(\right.$ Table $1^{1}$ ):

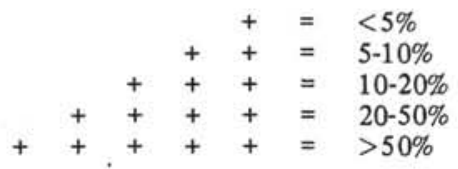

With the exception of the samples from the "evaporites" of Site 374 (Core 17 to Core 21), halite has not been listed though present since in most cases it

\footnotetext{
${ }^{1}$ A to abbreviations used in Table 1 appear on its last section.
}

formed during the drying of samples from the entrapped interstitial water. In some instances ("bomb", samples) bassanite $\left(\mathrm{CaSO}_{4} \times 1 / 2 \mathrm{H}_{2} \mathrm{O}\right)$ was noted. It is assumed that it originated by drying "bomb" samples containing gypsum at elevated temperatures $\left(>60^{\circ} \mathrm{C}\right)$ on shipboard. Bassanite was therefore listed as gypsum.

Since no oriented powder specimens were run, no attempt has been made to quantify clay minerals, they were only recorded (with the exception of mixed-layers and smectite) when distinct reflections were noted on the diffractogram.

In the sections between Cores 12 and 15 of Site 374 occur small white nodules which were identified as lüneburgite $\left[\mathrm{Mg}_{3}\left(\mathrm{PO}_{4}\right)_{2} \mathrm{~B}_{2} \mathrm{O}(\mathrm{OH})_{4} \times 6 \mathrm{H}_{2} \mathrm{O}\right.$ ], they are described in more detail by Müller and Fabricius (this volume).

X-ray diffractograms from Samples 374-11-2, 120$121 \mathrm{~cm}$, and $374-17-1,67-68 \mathrm{~cm}$, contain a number of reflections which were tentatively ascribed to bischoffite $\left(\mathrm{MgCl}_{2} \times 6 \mathrm{H}_{2} \mathrm{O}\right)$, the latter sample contains additional peaks which could not be identified, they are probably related to $\mathrm{MgSO}_{4}$.

\section{ACKNOWLEDGMENTS}

This study is a part of the sedimentological survey of Leg $42 \mathrm{~A}$ samples financed by the Deutsche Forschungsgemeinschaft. The help of W. Schuster, K. Lutz, and A. Schneider during the laboratory work is gratefully acknowledged. Thanks are also due to $\mathrm{R}$. McDuff for a review of the manuscript.

\section{REFERENCES}

Füchtbauer, H. and Goldschmidt, H., 1965. Beziehung zwischen Calciumgehalt und Bildungsbedingungen der Dolomite: Geol. Rundschau, v. 55, p. 29-40.

Goldsmith, J. R. and Graf, D. L., 1958. Relation between lattice constants and composition of $\mathrm{Ca}-\mathrm{Mg}$ carbonates. Am. Mineralogist, v. 43, p. 84-101.

Müller, G. and Müller, J., 1967. Mineralogisch-sedimentpetrographische und chemische Untersuchungen an einem Bank-Sediment (Cross Bank) der Florida Bay (USA): N.Jb. Miner. Abhandlungen, v. 106, p. 257-286.

Müller, J., 1969. Mineralogisch-sedimentpetrographische Utersuchungen an Karbonatsedimenten aus dem Schelfbereich um Fuerteventura und Lanzarote (Kanarische Inseln): Dissertation, University Heidelberg, p. 99.

Tennant, C. B. and Berger, R. W., 1957. X-ray determination of dolomite-calcite ratios of carbonate rocks: Am. Mineralogist, v. 42 , p. $23-29$. 
TABLE 1

Carbonate and Bulk Mineralogy of DSDP Leg 42A Samples

\begin{tabular}{|c|c|c|c|c|c|c|c|c|c|c|c|c|c|c|c|}
\hline $\begin{array}{c}\text { Sample } \\
\text { (Interval in } \mathrm{cm} \text { ) }\end{array}$ & $\begin{array}{l}\text { Tot. } \\
\text { Carb. }\end{array}$ & $\begin{array}{c}\text { Calc. } \\
(\%)\end{array}$ & $\underset{(\%)}{\mathrm{MgC} .}$ & $\begin{array}{l}\mathrm{Mol}-\% \\
\mathrm{MgCO}_{3}\end{array}$ & $\begin{array}{l}\text { Dol. } \\
(\%)\end{array}$ & $\begin{array}{l}\mathrm{Mol}-\% \\
\mathrm{Ca} / \mathrm{Mg}\end{array}$ & $\begin{array}{c}\text { Arga. } \\
(\%)\end{array}$ & $\begin{array}{c}\text { Magn. } \\
(\%)\end{array}$ & Quar. & K-Fel. & Plag. & Gyps. & Anhy. & Pyr. & Others \\
\hline \multicolumn{16}{|l|}{ Site 371} \\
\hline $1-1,100-150$ & 28 & 27 & - & - & 1 & $\begin{array}{l}50 / 50 \\
54 / 46\end{array}$ & - & - & +++ & - & + & - & - & - & IL, $\mathrm{CH}, \mathrm{KA}$ \\
\hline $4-2,13$ & $30^{\circ}$ & 29 & - & - & 1 & $\begin{array}{l}50 / 50 \\
54 / 46\end{array}$ & - & - & +++ & + & + & - & - & - & $\mathrm{IL}, \mathrm{CH}, \mathrm{KA}$ \\
\hline $4-2,15$ & 34 & 34 & - & - & - & - & - & - & +++ & + & + & - & - & - & IL, $\mathrm{CH}, \mathrm{KA}$ \\
\hline $4-2,130$ & 32 & 37 & - & - & 1 & $\begin{array}{l}50 / 50 \\
54 / 46\end{array}$ & - & - & +++ & - & + & - & - & - & IL, $\mathrm{CH}, \mathrm{KA}$ \\
\hline $8-2,116-118$ & 20 & - & - & - & 20 & $54 / 46$ & - & - & +t++ & + & ++ & - & - & - & $\mathrm{IL}, \mathrm{CH}, \mathrm{KA}$ \\
\hline $\begin{array}{l}8, \mathrm{CC} \\
\text { Site } 372\end{array}$ & 21 & 14 & - & - & 7 & $\begin{array}{l}50 / 50 \\
54 / 46\end{array}$ & - & - & ++++ & + & ++ & - & - & - & IL, $\mathrm{CH}, \mathrm{KA}$ \\
\hline $3-3,78-80$ & 43 & 39 & $+?$ & - & 4 & $\begin{array}{l}50 / 50 \\
54 / 46\end{array}$ & - & - & +++ & + & + & - & - & - & IL, $\mathrm{CH}$ \\
\hline $4-2,70-72$ & 41 & 37 & - & - & 4 & $\begin{array}{l}50 / 50 \\
54 / 46\end{array}$ & - & - & ++ & + & + & - & - & - & $\mathrm{IL}, \mathrm{CH}$ \\
\hline $\begin{array}{l}5-1,140 \\
6-1,140=141\end{array}$ & $\begin{array}{l}36 \\
21\end{array}$ & $\begin{array}{l}\text { N.D. } \rightarrow \\
\text { N.D. } \rightarrow\end{array}$ & & & & & & & & & & & & & \\
\hline $9-1,112-113(\mathrm{~A})$ & 27 & 26 & - & - & 1 & $\begin{array}{l}50 / 50 \\
54 / 46\end{array}$ & - & - & n+ & + & + & - & - & - & - \\
\hline $9-1,112-113(B)$ & 39 & 32 & - & - & 7 & $\begin{array}{l}50 / 50 \\
54 / 46\end{array}$ & - & - & ++ & + & ++ & - & - & - & IL, $\mathrm{CH}$ \\
\hline $9-3,10-12$ & 41 & 34 & - & - & 7 & $50 / 50$ & - & - & ++ & + & + & - & - & - & $\mathrm{IL}, \mathrm{CH}$ \\
\hline $12-4,134-135$ & 52 & 46 & - & - & 6 & $\begin{array}{l}50 / 50 \\
54 / 46\end{array}$ & - & - & ++ & + & + & - & - & - & $\mathrm{IL}, \mathrm{CH}$ \\
\hline $44, \mathrm{CC}$ & 57 & + & - & - & 57 & $54 / 46$ & - & - & +++ & - & + & - & - & - & IL, $\mathrm{CH}$ \\
\hline \multicolumn{16}{|l|}{ Hole 373A } \\
\hline $1-2,5-6$ & 22 & 21 & - & - & 1 & $\begin{array}{l}50 / 50 \\
54 / 46\end{array}$ & - & - & ++ & + & + & + & - & - & $\mathrm{IL}, \mathrm{CH}, \mathrm{KA}$ \\
\hline $1-2,12-14 \mathrm{~A}$ & 20 & 19 & - & - & 1 & $\begin{array}{l}50 / 50 \\
54 / 46\end{array}$ & - & - & ++ & + & + & + & - & - & IL, CH, KA \\
\hline $1-2,12-14 B$ & 28 & 27 & - & - & 1 & $50 / 50$ & - & - & ++ & + & + & - & - & - & IL, CH, KA \\
\hline $1-2,24-25$ & 32 & 31 & - & - & 1 & $\begin{array}{l}50 / 50 \\
54 / 46\end{array}$ & - & - & t+ & + & + & - & - & - & IL, CH, KA \\
\hline \multicolumn{16}{|l|}{ Site 374} \\
\hline $1-1,65-66$ & 49 & 10 & 18 & 12 & 9 & $\begin{array}{l}50 / 50 \\
54 / 46\end{array}$ & 12 & - & +++ & + & + & - & - & - & IL, CH, KA, HO? \\
\hline $1-1,123-124$ & 40 & 13 & 13 & 10 & 9 & $\begin{array}{l}50 / 50 \\
54 / 46\end{array}$ & 5 & - & +++ & + & ++ & - & - & - & IL, CH, KA, HO \\
\hline $1-1,136-138$ & 29 & 23 & 2 & 10 & 4 & $\begin{array}{l}50 / 50 \\
54 / 46\end{array}$ & + & - & +++ & + & ++ & - & - & - & $\mathrm{IL}, \mathrm{CH}, \mathrm{KA}, \mathrm{HO}$ \\
\hline $1-1,144-146$ & 43 & 23 & 4 & 12 & 9 & $\begin{array}{l}50 / 50 \\
54 / 46\end{array}$ & 7 & - & +++ & + & ++ & - & - & - & IL, CH, KA, HO? \\
\hline $1-2,17-19$ & 46 & 6 & 14 & 10 & 19 & $\begin{array}{l}50 / 50 \\
54 / 46\end{array}$ & 7 & - & ++++ & ++ & ++ & - & - & - & IL, CH, KA, HO? \\
\hline $1-2,145-147$ & 47 & 9 & 16 & 9 & 9 & $\begin{array}{l}50 / 50 \\
54 / 46\end{array}$ & 13 & - & ++++ & +++ & ++ & - & - & - & IL, CH, KA, HO? \\
\hline $1, \mathrm{CC}$ & 45 & 11 & 17 & 10 & 2 & $54 / 46$ & 15 & - & ++++ & +++ & t++ & - & - & - & IL, CH, KA, HO?, A? \\
\hline $2-1,57-59$ & 13 & 13 & - & - & + & - & - & - & +++ & + & ++ & - & - & - & IL, $\mathrm{CH}, \mathrm{KA}$ \\
\hline $2-1,121-123$ & 13 & 13 & - & - & + & - & - & - & +++ & + & + & - & - & - & $\mathrm{IL}, \mathrm{CH}, \mathrm{KA}$ \\
\hline $2-2,3-4$ & 15 & 15 & - & - & + & - & - & - & ++++ & ++ & +++ & - & - & - & IL, CH, KA \\
\hline $2-2,22-23$ & 17 & 17 & - & - & + & - & - & - & +++ & + & ++ & - & - & - & $\mathrm{IL}, \mathrm{CH}, \mathrm{KA}$ \\
\hline $2-3,21-22$ & 20 & 18 & - & - & 2 & $50 / 50$ & - & - & +++ & + & ++ & - & - & - & IL, CH, KA \\
\hline $2-3,123-124$ & 28 & 28 & - & - & + & - & - & - & ++ & + & + & + & - & + & $\mathrm{IL}, \mathrm{CH}, \mathrm{KA}$ \\
\hline $2, C C(A)$ & 44 & 14 & 12 & 9 & 11 & $50 / 50$ & 7 & - & +++ & ++ & + & - & - & - & IL \\
\hline 2, CC (B) & 38 & 37 & - & - & 1 & - & - & - & ++ & + & + & + & - & + & IL, $\mathrm{CH}, \mathrm{KA}$ \\
\hline $2, \mathrm{CC}(\mathrm{C})$ & 22 & 20 & - & - & 2 & $\begin{array}{l}50 / 50 \\
54 / 46\end{array}$ & - & - & +++ & + & + & - & - & - & $\mathrm{IL}, \mathrm{CH}, \mathrm{KA}$ \\
\hline $3-1,103-104$ & 33 & 33 & - & - & + & - & - & - & t+ & + & + & + & - & + & IL \\
\hline $3-1,104-105$ & 45 & 45 & - & - & + & - & - & - & ++ & + & + & + & - & + & IL, $\mathrm{CH}, \mathrm{KA}$ \\
\hline $3-1,122-123$ & 34 & 31 & - & - & 3 & $50 / 50$ & - & - & ++ & + & + & + & - & + & IL, $\mathrm{CH}, \mathrm{KA}$ \\
\hline $3-1,126-127$ & 37 & 33 & - & - & 4 & $50 / 50$ & - & - & +++ & + & ++ & + & - & + & IL, CH, KA, HO \\
\hline $3-1,127-128$ & 39 & 36 & - & - & 3 & $50 / 50$ & - & - & ++ & + & + & + & - & + & $\mathrm{IL}, \mathrm{CH}, \mathrm{KA}$ \\
\hline $3-1,129-130$ & 15 & 15 & - & - & + & - & - & - & ++ & + & ++ & + & - & + & IL, $\mathrm{CH}, \mathrm{KA}$ \\
\hline $3-1,141-142$ & 23 & 23 & - & - & + & - & - & - & +++ & + & ++ & + & - & + & IL, $\mathrm{CH}, \mathrm{KA}$ \\
\hline $3-1,143-144$ & 19 & 15 & - & - & 4 & $50 / 50$ & - & - & +++ & + & ++ & + & - & + & $\mathrm{IL}, \mathrm{CH}, \mathrm{KA}$ \\
\hline $3, \mathrm{CC}$ & 23 & 19 & - & - & 4 & $50 / 50$ & - & - & +++ & + & ++ & - & - & - & IL, $\mathrm{CH}, \mathrm{KA}$ \\
\hline $4-2,55-56$ & 15 & 13 & - & - & 2 & $\begin{array}{l}50 / 50 \\
54 / 46\end{array}$ & - & - & +++ & + & ++ & - & - & - & $\mathrm{IL}, \mathrm{CH}, \mathrm{KA}$ \\
\hline $4-2,56-57$ & 38 & 38 & - & - & + & - & - & - & ++ & + & + & + & - & + & $\mathrm{IL}, \mathrm{CH}, \mathrm{KA}$ \\
\hline $4-2,57-58$ & 41 & 41 & - & - & - & - & - & - & ++ & + & + & + & - & + & IL, CH, KA \\
\hline $4-2,58-59$ & 49 & 49 & - & - & - & - & - & - & ++ & + & + & + & - & + & $\mathrm{IL}, \mathrm{CH}, \mathrm{KA}$ \\
\hline $4-2,59.5-60.5$ & 30 & 30 & - & - & + & - & - & - & t++ & + & + & + & - & + & IL, CH, KA \\
\hline
\end{tabular}


TABLE 1 - Continued

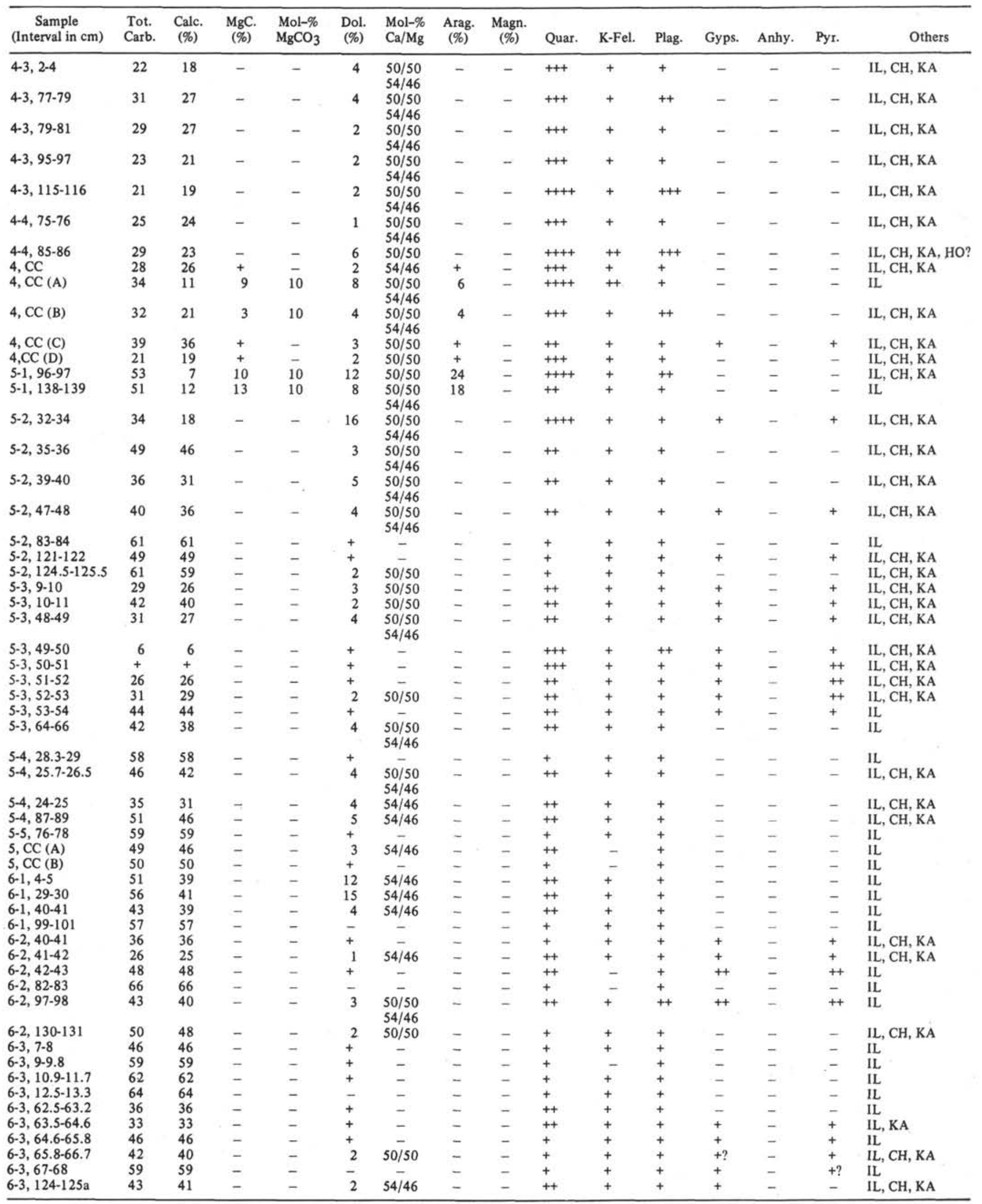


TABLE 1 - Continued

\begin{tabular}{|c|c|c|c|c|c|c|c|c|c|c|c|c|c|c|c|}
\hline $\begin{array}{c}\text { Sample } \\
\text { (Interval in } \mathrm{cm} \text { ) }\end{array}$ & $\begin{array}{l}\text { Tot. } \\
\text { Carb. }\end{array}$ & $\begin{array}{l}\text { Calc. } \\
(\%)\end{array}$ & $\underset{(\%)}{\mathrm{MgC} .}$ & $\begin{array}{l}\mathrm{Mol}-\% \\
\mathrm{MgCO}_{3}\end{array}$ & $\begin{array}{l}\text { Dol. } \\
(\%)\end{array}$ & $\begin{array}{l}\mathrm{Mol}-\% \\
\mathrm{Ca} / \mathrm{Mg}\end{array}$ & $\begin{array}{c}\text { Arag. } \\
(\%)\end{array}$ & $\begin{array}{l}\text { Magn. } \\
(\%)\end{array}$ & Quar. & K-Fel. & Plag. & Gyps. & Anhy. & Pyr. & Others \\
\hline $6-3,124-125 b$ & 29 & 27 & - & - & 2 & $54 / 46$ & - & - & t+ & + & + & + & - & + & IL, $\mathrm{CH}, \mathrm{KA}$ \\
\hline $6-3,125-126$ & 49 & 49 & - & - & + & - & - & - & + & + & + & + & - & + & IL \\
\hline $6-4,99-100$ & 60 & 60 & - & - & + & - & - & - & + & - & + & - & - & - & IL \\
\hline $6-5,40-42$ & 56 & 56 & - & - & + & - & - & - & + & + & + & - & - & - & IL, $\mathrm{CH}, \mathrm{KA}$ \\
\hline $6-5,46-48$ & 59 & 55 & - & - & 4 & $\begin{array}{l}50 / 50 \\
54 / 46\end{array}$ & - & - & ++ & + & ++ & - & - & - & IL, CH, KA \\
\hline $6-5,57-59$ & 56 & 56 & - & - & + & - & - & - & + & + & + & - & - & - & IL \\
\hline $6-5,63-65$ & 60 & 60 & - & - & + & - & - & - & + & + & + & - & - & - & $\mathrm{IL}, \mathrm{CH}, \mathrm{KA}$ \\
\hline $6-5,67-68$ & 56 & 47 & - & - & 9 & $\begin{array}{l}50 / 50 \\
54 / 46\end{array}$ & -. & - & ++ & + & - & - & - & - & IL \\
\hline $6-5,68-69$ & 56 & 51 & - & - & 5 & $\begin{array}{l}50 / 50 \\
54 / 46\end{array}$ & - & - & + & + & + & - & - & - & IL \\
\hline $6-5,69-70$ & 55 & 47 & - & - & 8 & $\begin{array}{l}50 / 50 \\
54 / 46\end{array}$ & - & - & ++ & + & + & - & - & - & IL \\
\hline $6-5,70-71$ & 62 & 54 & - & - & 8 & $54 / 46$ & - & - & ++ & + & + & - & - & - & IL \\
\hline $6-6,10.7-11.3$ & 58 & 54 & - & - & 4 & $\begin{array}{l}50 / 50 \\
54 / 46\end{array}$ & - & - & + & - & + & - & - & - & IL \\
\hline $6-6,11.3-12.4$ & 44 & 40 & - & - & 4 & $\begin{array}{l}50 / 50 \\
54 / 46\end{array}$ & - & - & ++ & - & + & - & - & - & IL \\
\hline $6-6,14-15.2$ & 69 & 63 & - & - & 6 & $\begin{array}{l}50 / 50 \\
54 / 46\end{array}$ & - & - & ++ & + & + & - & - & - & IL \\
\hline $6-6,15.2-16$ & 65 & 65 & - & - & + & - & - & - & + & - & + & - & - & - & IL \\
\hline $6-6,84-86$ & 59 & 59 & - & - & + & - & - & - & + & - & + & - & - & - & IL, KA \\
\hline $6, \mathrm{CC}(\mathrm{A})$ & 62 & 55 & - & - & 7 & $54 / 46$ & - & - & + & + & + & - & - & - & IL \\
\hline $6, \mathrm{CC}$ (B) & 59 & 59 & - & - & + & - & - & - & + & + & + & - & - & - & IL \\
\hline $7-1,83-88$ & 46 & 41 & - & - & 5 & $54 / 46$ & - & - & + & + & + & - & - & - & IL \\
\hline $7-2,68-73$ & 46 & 41 & - & - & 5 & $54 / 46$ & - & - & + & + & + & - & - & - & IL \\
\hline $7-3,72-77$ & 44 & 38 & - & - & 6 & $54 / 46$ & - & - & + & + & + & - & - & - & IL \\
\hline $7-4,83-88$ & 41 & 36 & - & - & 5 & $\begin{array}{l}50 / 50 \\
54 / 46\end{array}$ & - & - & + & + & + & - & - & - & IL \\
\hline $7-5,53-58$ & 59 & 53 & - & - & 6 & $54 / 46$ & - & - & + & + & + & - & - & - & IL \\
\hline $7-5,71-76$ & 52 & 44 & - & - & 8 & $\begin{array}{l}50 / 50 \\
54 / 46\end{array}$ & - & - & + & + & + & - & - & - & IL \\
\hline $7-6,52-57$ & 54 & 48 & - & - & 6 & $54 / 46$ & - & - & + & + & + & - & - & - & IL \\
\hline $8-1,115-116$ & 67 & 55 & - & - & 12 & $54 / 46$ & - & - & + & + & + & - & - & - & IL \\
\hline $8-2,24-26$ & 52 & 43 & - & - & 9 & $\begin{array}{l}50 / 50 \\
54 / 46\end{array}$ & - & - & ++ & + & + & - & - & - & IL, CH, KA \\
\hline $8-2,63-64$ & 62 & 55 & - & - & 7 & $54 / 46$ & - & - & + & - & + & - & - & - & IL \\
\hline $8-3,98-99$ & 62 & 56 & - & - & 6 & $54 / 46$ & - & - & + & - & + & - & - & - & IL \\
\hline $9-1,150-151$ & 53 & 50 & - & - & 3 & $54 / 46$ & - & - & + & + & + & - & - & - & IL \\
\hline $9-3,15-16$ & 63 & 59 & - & - & 4 & $54 / 46$ & - & - & + & - & + & - & - & - & IL \\
\hline $9-3,72-77$ & 67 & 60 & - & - & 7 & $54 / 46$ & - & - & + & - & + & - & - & - & IL \\
\hline $11-2,112$ & 79 & - & - & - & 79 & $55 / 45$ & - & - & + & - & + & - & - & - & $\mathrm{IL}, \mathrm{CH}, \mathrm{KA}$ \\
\hline $11-2,120-121$ & 78 & - & - & - & 78 & $55 / 45$ & - & - & + & - & - & - & - & - & IL, BI? \\
\hline $11-2,147-149$ & 75 & - & - & - & 75 & $55 / 45$ & - & - & + & + & + & - & - & + & $\mathrm{IL}, \mathrm{CH}, \mathrm{KA}$ \\
\hline $11, \mathrm{CC}(\mathrm{A})$ & 77 & - & - & - & 77 & $55 / 45$ & - & - & + & + & + & - & - & + & IL \\
\hline $11, \mathrm{CC}(\mathrm{B})$ & 78 & - & - & - & 78 & $55 / 45$ & - & - & + & + & + & - & - & + & IL \\
\hline $11, \mathrm{CC}(\mathrm{C})$ & 69 & - & - & - & 69 & $55 / 45$ & - & - & + & - & + & - & - & $+?$ & IL, KA \\
\hline $11, C C$ (D) & n.d. & - & - & - & - & - & - & - & - & - & - & - & - & +++++ & - \\
\hline $12-2,99-100$ & 21 & - & - & - & 21 & $55 / 45$ & - & - & t++ & + & + & +++ & - & $+?$ & - \\
\hline $13-1,133-135$ & 20 & - & - & - & 20 & $55 / 45$ & - & - & +++ & + & + & - & - & - & $\mathrm{IL}, \mathrm{L}$ \\
\hline $\begin{array}{l}\text { 14-1, TOP- } \\
\text { WASTE }\end{array}$ & 21 & - & - & - & 21 & $55 / 45$ & - & - & +++ & + & ++ & - & - & - & IL, CH, KA \\
\hline $14-1,100-102$ & 19 & - & - & - & 19 & $55 / 45$ & - & - & +++ & + & t+ & - & - & - & IL, CH, KA, L \\
\hline $15-1,69-70$ & 25 & - & - & - & 25 & $55 / 45$ & - & - & +++ & + & ++ & - & - & + ? & $\mathrm{IL}, \mathrm{CH}, \mathrm{KA}, \mathrm{L}$ \\
\hline $15-1,100-102$ & 24 & - & - & - & 24 & $55 / 45$ & - & - & +++ & + & ++ & - & - & $+?$ & IL, CH, KA, L \\
\hline $15-2,79-81$ & 23 & - & - & - & 23 & $55 / 45$ & - & - & +++ & + & ++ & - & - & + ? & IL, CH, KA \\
\hline $17-1,62-63$ & 16 & + ? & - & - & 16 & $55 / 45$ & - & - & + & - & - & ++++ & - & - & IL, HA \\
\hline $17-1,67-68$ & 36 & - & - & - & 36 & $55 / 45$ & - & + & ++ & - & - & ++ & - & - & IL, Bl?, HA, U \\
\hline $17-1,80-81$ & 37 & $+?$ & - & - & 37 & $55 / 45$ & - & - & + & - & - & ++++ & ++ & - & IL, HA \\
\hline $19-1,28-29$ & 51 & + ? & - & - & 51 & $55 / 45$ & - & $+?$ & + & - & - & +++ & t+ & - & IL, HA \\
\hline $19-1,52-53$ & 28 & - & - & - & 28 & $55 / 45$ & - & - & ++ & - & - & +++ & + & - & IL, HA \\
\hline $20-1,24-25$ & 11 & - & - & - & 11 & $55 / 45$ & - & - & ++ & - & $+?$ & +++ & + & + & $\mathrm{IL}, \mathrm{HA}, \mathrm{KA}, \mathrm{CH}$ \\
\hline $21-1,105-108$ & 22 & - & - & - & - & - & - & 22 & + & - & - & - & ++++ & - & $\mathrm{HA}$ \\
\hline $\begin{array}{l}\text { 25-SIDE } \\
\text { WALL } 1\end{array}$ & 71 & - & - & - & 71 & $55 / 45$ & - & - & + & - & + & - & - & - & IL \\
\hline $\begin{array}{l}\text { 25-SIDE } \\
\text { WALL } 2\end{array}$ & N.D. & ++++ & - & - & +++ & $55 / 45$ & - & - & + & - & - & - & - & - & IL, BI? \\
\hline $\begin{array}{l}\text { 26-SIDE } \\
\text { WALL }\end{array}$ & 64 & 51 & - & - & 13 & $55 / 45$ & - & - & + & - & + & ++ & - & - & IL \\
\hline \multicolumn{16}{|l|}{ Site 375} \\
\hline $1-1,50-51$ & 37 & 36 & - & - & 1 & $54 / 46$ & - & - & ++ & + & + & - & - & - & - \\
\hline $1-1,123-125$ & 23 & 18 & - & - & 5 & $\begin{array}{l}50 / 50 \\
54 / 46\end{array}$ & - & - & +++ & + & ++ & + & - & - & - \\
\hline
\end{tabular}


TABLE 1 - Continued

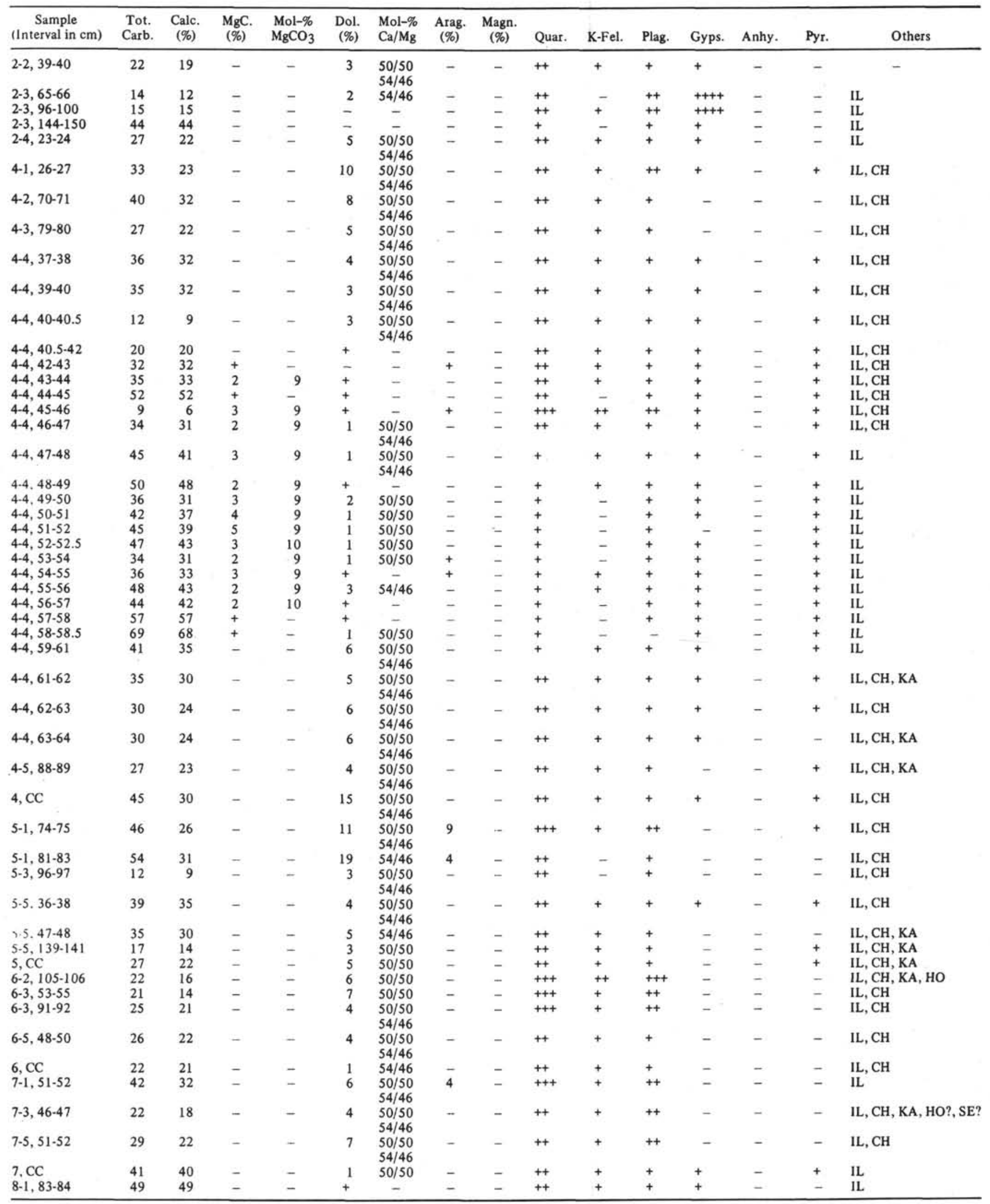


TABLE 1 - Continued

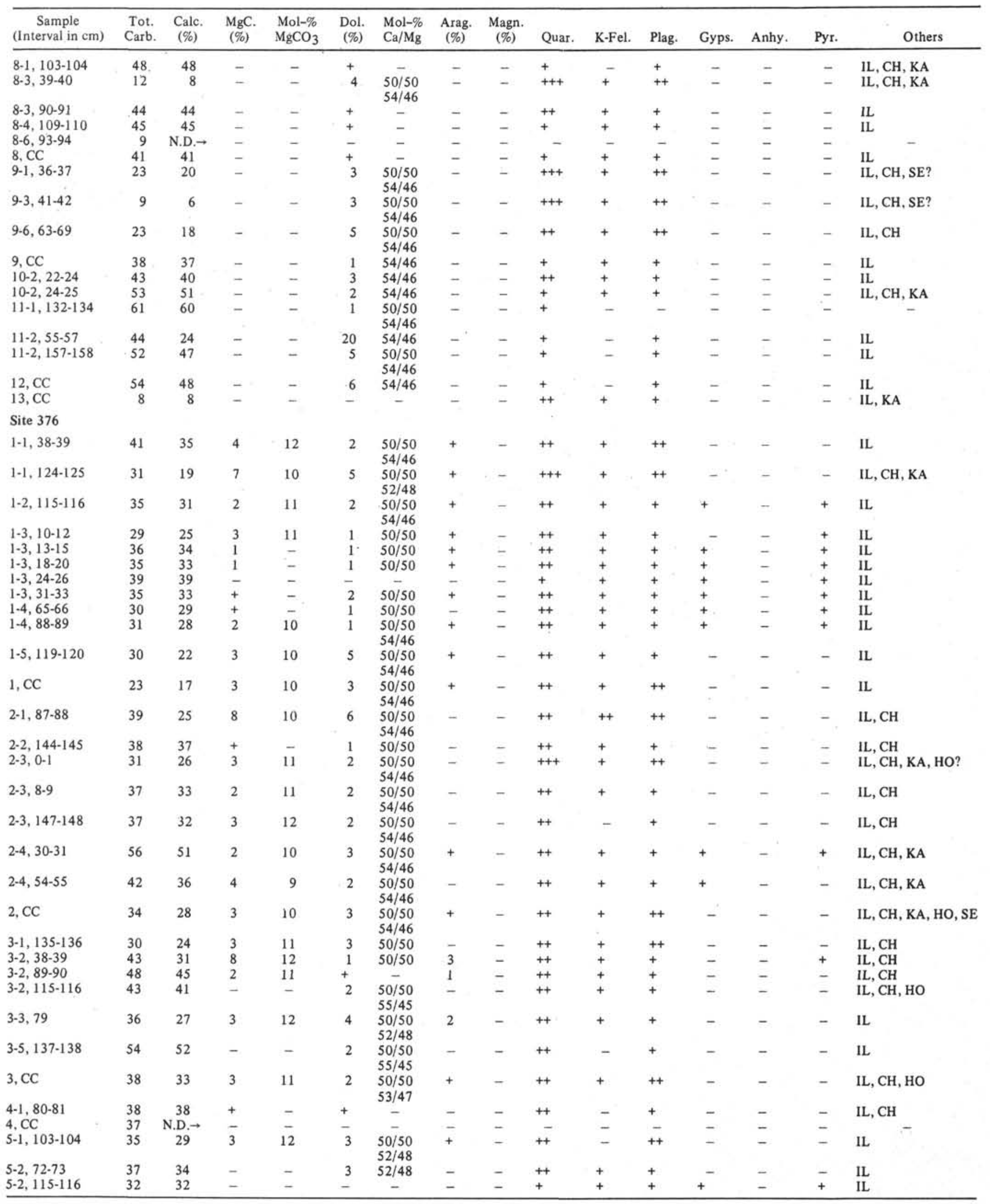


TABLE 1 - Continued

\begin{tabular}{|c|c|c|c|c|c|c|c|c|c|c|c|c|c|c|c|}
\hline $\begin{array}{c}\begin{array}{c}\text { Sample } \\
\text { (Interval in } \mathrm{cm} \text { ) }\end{array} \\
\end{array}$ & $\begin{array}{l}\text { Tot. } \\
\text { Carb. }\end{array}$ & $\begin{array}{l}\text { Calc. } \\
(\%)\end{array}$ & $\begin{array}{l}\mathrm{MgC}_{(\%)} \\
(\%)\end{array}$ & $\begin{array}{l}\mathrm{Mol}-\% \\
\mathrm{MgCO}_{3}\end{array}$ & $\begin{array}{l}\text { Dol. } \\
(\%)\end{array}$ & $\begin{array}{l}\mathrm{Mol}-\% \\
\mathrm{Ca} / \mathrm{Mg}\end{array}$ & $\begin{array}{l}\text { Arag. } \\
(\%)\end{array}$ & $\begin{array}{c}\text { Magn. } \\
(\%)\end{array}$ & Quar. & K-Fel. & Plag. & Gyps. & Anhy. & Pyr. & Others \\
\hline $5-3,60-61$ & 51 & 47 & - & - & 4 & $\begin{array}{l}50 / 50 \\
55 / 45\end{array}$ & - & - & ++ & - & + & - & - & - & IL \\
\hline $5-4,38-39$ & 48 & 45 & - & - & 3 & $\begin{array}{l}50 / 50 \\
54 / 46\end{array}$ & - & - & + & + & + & - & - & - & IL \\
\hline $5-4,64-65$ & 43 & 40 & - & - & 3 & $\begin{array}{l}50 / 50 \\
55 / 45\end{array}$ & - & - & ++ & + & + & - & - & - & IL \\
\hline $5-4,105-106$ & 28 & 28 & - & - & + & 5 & - & - & + & + & + & + & - & + & IL \\
\hline $\begin{array}{l}5-5,95-96 \\
5, \text { CC }\end{array}$ & $\begin{array}{l}28 \\
36\end{array}$ & $\begin{array}{l}26 \\
17\end{array}$ & $\bar{z}$ & $\overline{-}$ & $\begin{array}{r}2 \\
19\end{array}$ & $\begin{array}{l}54 / 46 \\
50 / 50\end{array}$ & $\overline{-}$ & $\overline{-}$ & $\begin{array}{l}++ \\
++\end{array}$ & $\begin{array}{l}+ \\
++\end{array}$ & $\begin{array}{l}+ \\
++\end{array}$ & $\overline{-}$ & - & - & IL $\mathrm{CH}$ но \\
\hline $6-1,133-134$ & 36 & 34 & - & - & 2 & $\begin{array}{l}55 / 45 \\
50 / 50\end{array}$ & - & - & ++ & $+\tau$ & + & - & - & - & \\
\hline $6-2,83-84$ & 42 & 37 & - & - & 5 & $\begin{array}{l}54 / 46 \\
50 / 50\end{array}$ & - & - & ++ & + & + & - & - & - & $\mathrm{IL}, \mathrm{CH}, \mathrm{KA}$ \\
\hline $6-3,94-95$ & 9 & 9 & - & - & + & $55 / 45$ & - & & ++ & + & + & - & - & - & $\mathrm{IL}, \mathrm{CH}, \mathrm{HO}$ \\
\hline $6-4,50-51$ & 64 & 57 & - & - & 7 & $55 / 45$ & $\overline{-}$ & $\overline{-}$ & + & + & + & $\overline{-}$ & $\overline{-}$ & $\overline{-}$ & $\begin{array}{l}\text { IL, CH, KA } \\
\text { IL }\end{array}$ \\
\hline $6-4,56-57$ & 57 & 50 & - & - & 7 & $55 / 45$ & - & - & + & - & + & 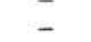 & - & 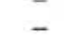 & IL \\
\hline $6-4,57-58$ & 58 & 52 & - & - & 6 & $55 / 45$ & - & - & + & - & + & + & - & - & IL \\
\hline $6-4,58-59$ & 64 & 58 & - & - & 6 & $55 / 45$ & - & - & + & - & + & + & - & + & IL \\
\hline $6-4,60-61$ & 63 & 59 & - & - & 4 & $54 / 46$ & - & - & + & + & + & + & - & + & IL, $\mathrm{CH}$ \\
\hline $6-4,61-62$ & 68 & 61 & - & - & $?$ & $54 / 46$ & - & - & + & + & + & + & - & + & IL \\
\hline $6-4,63-64$ & 51 & 45 & - & - & 6 & $54 / 46$ & - & - & + & + & + & - & - & + & IL \\
\hline $6-4,64-65$ & 57 & 50 & - & - & 7 & $\begin{array}{l}50 / 50 \\
54 / 46\end{array}$ & - & - & + & + & + & + & - & - & IL \\
\hline $6-4,66-67$ & 51 & 45 & - & - & 6 & $54 / 46$ & - & - & + & + & + & + & - & + & IL \\
\hline $\begin{array}{l}6-4,68-69 \\
6-4,70-71\end{array}$ & 50 & 43 & - & - & 7 & $54 / 46$ & - & - & + & + & + & + & - & + & IL, CH, KA \\
\hline $\begin{array}{l}6-4,70-71 \\
6-4,72-73\end{array}$ & 60 & 54 & - & - & 6 & $54 / 46$ & - & - & + & + & + & + & - & + & \\
\hline $\begin{array}{l}6-4,72-73 \\
6-4,74-75\end{array}$ & 62 & 57 & - & - & 5 & $54 / 46$ & - & - & + & + & + & + & - & + & IL \\
\hline $\begin{array}{l}6-4,74-75 \\
6-4,75-76\end{array}$ & 77 & 71 & - & - & 6 & $54 / 46$ & - & - & + & + & + & + & - & + & IL, CH \\
\hline $\begin{array}{l}6-4,75-76 \\
6-4,77-78\end{array}$ & 68 & 64 & - & - & 4 & $54 / 46$ & - & - & + & + & + & - & - & - & IL, CH, KA \\
\hline $6-4,77-78$ & 63 & 57 & - & - & 6 & $\begin{array}{l}50 / 50 \\
54 / 46\end{array}$ & - & - & + & - & + & + & - & + & IL \\
\hline $\begin{array}{r}6-4,78-79 \\
6-4,81-82\end{array}$ & 59 & 51 & - & - & 8 & $54 / 46$ & - & - & + & + & + & - & - & - & IL \\
\hline $\begin{array}{l}6-4,81-82 \\
7-1,59-60\end{array}$ & $\begin{array}{l}61 \\
29\end{array}$ & 52 & $=$ & - & 9 & $\begin{array}{l}54 / 46 \\
50 / 50\end{array}$ & - & - & + & - & - & + & - & - & IL \\
\hline & 29 & 10 & - & & 19 & $\begin{array}{l}50 / 50 \\
54 / 46\end{array}$ & - & - & ++ & + & ++ & - & - & - & IL \\
\hline $7-2,51-52$ & 30 & 10 & - & - & 20 & $\begin{array}{l}50 / 50 \\
54 / 46\end{array}$ & - & - & ++ & + & ++ & - & - & - & $\mathrm{IL}, \mathrm{CH}, \mathrm{KA}$ \\
\hline $7, \mathrm{CC}$ & 35 & 22 & - & - & 13 & $\begin{array}{l}54 / 46 \\
50 / 50\end{array}$ & - & - & ++ & + & ++ & - & - & - & IL, CH, HO \\
\hline $8-1,140-141$ & 31 & 20 & - & - & 11 & $\begin{array}{l}54 / 46 \\
50 / 50\end{array}$ & - & - & ++ & + & ++ & - & - & - & IL, CH, HO \\
\hline $8-2,69-70$ & 41 & 24 & - & - & 17 & $\begin{array}{l}54 / 46 \\
50 / 50 \\
54 / 46\end{array}$ & - & - & ++ & + & ++ & - & - & - & IL, $\mathrm{CH}$ \\
\hline $8-3,17-18$ & 33 & 21 & - & - & 12 & $\begin{array}{l}50 / 50 \\
54 / 46\end{array}$ & - & - & ++ & + & ++ & - & - & - & IL, $\mathrm{CH}, \mathrm{KA}$ \\
\hline $8-3,31-32$ & 32 & 24 & - & - & 8 & $\begin{array}{l}50 / 50 \\
54 / 46\end{array}$ & - & - & ++ & + & ++ & - & - & - & IL \\
\hline $8-3,36-37$ & 28 & 15 & - & - & 13 & $\begin{array}{l}50 / 50 \\
54 / 46\end{array}$ & - & - & ++ & ++ & ++ & - & - & - & IL, $\mathrm{CH}, \mathrm{HO}$ \\
\hline $8, \mathrm{CC}$ & 37 & N.D. $\rightarrow$ & - & - & - & - & - & - & - & - & - & - & - & - & - \\
\hline $9-1,50-51$ & 35 & 18 & - & - & 17 & $\begin{array}{l}50 / 50 \\
54 / 46\end{array}$ & - & - & +++ & ++ & ++ & - & - & - & IL, CH \\
\hline $9-1,81-83$ & 35 & 27 & - & - & 8 & $\begin{array}{l}50 / 50 \\
54 / 46\end{array}$ & - & - & ++ & + & + & - & - & - & IL, $\mathrm{CH}$ \\
\hline $9-2,70-71$ & 29 & 23 & - & - & 6 & $54 / 46$ & - & - & ++ & + & + & - & - & - & IL, CH \\
\hline $\begin{array}{l}9-3,70-71 \\
9-4,4-5\end{array}$ & 29 & 25 & - & - & 4 & $54 / 46$ & - & - & +++ & ++ & ++ & - & - & - & IL, $\mathrm{CH}, \mathrm{HO}$ \\
\hline $9-4,4-5$ & 36 & 25 & - & - & 11 & $\begin{array}{l}50 / 50 \\
54 / 46\end{array}$ & - & - & ++ & + & ++ & - & - & - & $\mathrm{IL}, \mathrm{CH}$ \\
\hline $9-4,80-81$ & 38 & 19 & - & - & 19 & $\begin{array}{l}50 / 50 \\
54 / 46\end{array}$ & - & - & +++ & + & +++ & - & - & - & IL, CH \\
\hline $9, \mathrm{CC}$ & 28 & 24 & - & - & 4 & $\begin{array}{l}50 / 50 \\
54 / 46\end{array}$ & - & - & ++ & + & + & - & - & - & IL, HO \\
\hline $10-1,101-102$ & 39 & 27 & - & - & 12 & $\begin{array}{l}50 / 50 \\
54 / 46\end{array}$ & - & - & +++ & + & ++ & - & - & - & IL, $\mathrm{CH}$ \\
\hline $10-3,100-101$ & 35 & 27 & - & - & 8 & $\begin{array}{l}50 / 50 \\
55 / 46\end{array}$ & - & - & ++ & + & + & - & - & - & $\mathrm{IL}, \mathrm{CH}, \mathrm{KA}$ \\
\hline $10, \mathrm{CC}$ & 37 & 27 & - & - & 10 & $\begin{array}{l}54 / 46 \\
50 / 50\end{array}$ & - & - & t+ & + & ++ & - & - & - & IL, CH, KA \\
\hline $11-1,139-140$ & 39 & 26 & - & - & 13 & $\begin{array}{l}54 / 46 \\
50 / 50\end{array}$ & - & - & ++ & + & ++ & - & - & - & IL, $\mathrm{CH}$ \\
\hline $11-2,130-131$ & 71 & 71 & - & - & + & $\begin{array}{c}54 / 46 \\
-\end{array}$ & - & - & + & + & + & - & - & - & IL \\
\hline $11-2,133-136$ & 45 & 45 & - & - & - & - & - & - & + & - & - & - & - & - & IL \\
\hline $11, \mathrm{CC}$ & 70 & 69 & - & - & 1 & $55 / 45$ & - & - & + & + & + & - & - & - & IL \\
\hline $12-1,22-28$ & 60 & 59 & - & - & 1 & $55 / 45$ & - & - & + & + & + & - & - & - & $\mathrm{IL}, \mathrm{CH}$ \\
\hline
\end{tabular}


TABLE 1 - Continued

\begin{tabular}{|c|c|c|c|c|c|c|c|c|c|c|c|c|c|c|c|}
\hline $\begin{array}{c}\text { Sample } \\
\text { (Interval in } \mathrm{cm} \text { ) }\end{array}$ & $\begin{array}{l}\text { Tot. } \\
\text { Carb. }\end{array}$ & $\begin{array}{l}\text { Calc. } \\
(\%)\end{array}$ & $\underset{(\%)}{\mathrm{MgC} .}$ & $\begin{array}{l}\mathrm{Mol}-\% \\
\mathrm{MgCO}_{3}\end{array}$ & $\begin{array}{l}\text { Dol. } \\
(\%)\end{array}$ & $\begin{array}{l}\mathrm{Mol}-\% \\
\mathrm{Ca} / \mathrm{Mg}\end{array}$ & $\begin{array}{l}\text { Arag. } \\
(\%)\end{array}$ & $\begin{array}{l}\text { Magn. } \\
(\%)\end{array}$ & Quar. & K-Fel. & Plag. & Gyps. & Anhy. & Pyr. & Others \\
\hline $12-1,40-42$ & 37 & 37 & - & - & + & - & - & - & ++ & + & + & - & - & - & IL \\
\hline $12-2,49-51$ & 36 & 35 & - & - & 1 & $55 / 45$ & - & - & + & + & + & + & - & - & $\mathrm{IL}, \mathrm{CH}$ \\
\hline $12-2,70-71$ & 23 & N.D. $\rightarrow$ & - & - & - & - & - & - & - & - & - & - & - & - & - \\
\hline $12-2,90-91$ & 37 & 33 & - & - & 4 & $\begin{array}{l}50 / 50 \\
54 / 46\end{array}$ & - & - & + & + & + & - & - & + & $\mathrm{IL}, \mathrm{CH}$ \\
\hline $12-3,130-131$ & 56 & 53 & - & - & 3 & $\begin{array}{l}50 / 50 \\
54 / 46\end{array}$ & - & - & + & - & + & - & - & - & IL \\
\hline $12-4,70-71$ & 23 & 23 & - & - & + & - & - & - & ++ & + & + & - & - & - & IL \\
\hline $12-5,130-131$ & 38 & 38 & - & - & + & - & - & - & ++ & + & ++ & - & - & - & IL \\
\hline $12, \mathrm{CC}$ & 39 & 39 & - & - & + & - & - & - & ++ & + & + & - & - & - & IL \\
\hline $13-1,114-115$ & 66 & 66 & - & - & + & - & - & - & + & - & + & - & - & - & IL \\
\hline $13-2,71-72$ & 32 & 32 & - & - & + & - & - & - & ++ & + & + & - & - & - & IL \\
\hline $13-4,101-102$ & 25 & 25 & - & - & + & - & - & - & ++ & + & ++ & - & - & - & IL, CH \\
\hline $13, \mathrm{CC}$ & 21 & 21 & - & - & + & - & - & - & +++ & + & ++ & - & - & - & IL, $\mathrm{CH}$ \\
\hline $14, \mathrm{CC}$ & 24 & 24 & - & - & + & - & - & - & +++ & + & ++ & - & - & - & IL \\
\hline $15-1,137-138$ & 45 & 45 & - & - & - & - & - & - & + & + & + & - & - & - & IL \\
\hline $15-2,35-36$ & 47 & 47 & - & - & - & - & - & - & + & - & - & +++ & - & - & IL \\
\hline $15-2,125-126$ & 36 & 29 & - & - & 7 & $\begin{array}{l}50 / 50 \\
52 / 48\end{array}$ & - & - & ++ & + & + & - & - & - & IL \\
\hline $15-3,2-3$ & 44 & 41 & - & - & 3 & $\begin{array}{l}50 / 50 \\
52 / 48\end{array}$ & - & - & ++ & + & + & - & - & - & $\mathrm{IL}, \mathrm{CH}$ \\
\hline $15, \mathrm{CC}$ & 41 & 41 & - & - & + & - & - & - & ++ & + & + & +++ & $-\cdot$ & - & IL \\
\hline $17-1,45-47$ & 37 & 36 & - & - & 1 & $54 / 46$ & - & - & + & - & $+?$ & +++ & - & - & - \\
\hline \multicolumn{16}{|l|}{ Site 377} \\
\hline $1-1,65-67$ & 51 & 47 & 3 & 10 & 1 & $\begin{array}{l}50 / 50 \\
54 / 46\end{array}$ & + & - & ++ & + & + & - & - & - & $\mathrm{IL}, \mathrm{CH}, \mathrm{KA}$ \\
\hline $1-1,104-106$ & 68 & 63 & 4 & 10 & 1 & $\begin{array}{l}50 / 50 \\
54 / 46\end{array}$ & + & $-\cdot$ & ++ & + & + & - & - & - & IL \\
\hline $1-1,149-150$ & 50 & 49 & - & - & 1 & $\begin{array}{l}50 / 50 \\
54 / 46\end{array}$ & - & - & ++ & + & + & + & - & + & IL \\
\hline $1-2,10-11$ & 34 & 32 & - & - & 2 & $\begin{array}{l}50 / 50 \\
54 / 46\end{array}$ & - & - & ++ & + & + & - & - & + & $\mathrm{IL}, \mathrm{CH}, \mathrm{KA}$ \\
\hline $1-2,28-29$ & 36 & 25 & - & - & 11 & $\begin{array}{l}50 / 50 \\
54 / 46\end{array}$ & - & - & ++ & + & + & - & - & + & IL \\
\hline $1-2,97-98$ & 46 & 42 & 3 & 10 & 1 & $\begin{array}{l}50 / 50 \\
54 / 46\end{array}$ & - & - & ++ & + & + & + & - & ++ & IL \\
\hline $1-2,109-111$ & 50 & 45 & 4 & 9 & 1 & $\begin{array}{l}50 / 50 \\
54 / 46\end{array}$ & $+?$ & - & ++ & + & + & - & - & - & IL \\
\hline $2-1,105-106$ & 52 & 51 & - & - & 1 & $\begin{array}{l}50 / 50 \\
54 / 46\end{array}$ & - & - & ++ & + & + & - & - & - & IL, CH, KA \\
\hline $3-2,19-23$ & 23 & 23 & - & - & - & - & - & - & +++ & + & + & - & - & - & IL, CH, KA \\
\hline $4-3,140-142$ & 13 & 13 & - & - & + & - & - & - & +++ & + & + & + & - & + & IL, CH, KA \\
\hline \multicolumn{16}{|l|}{ Hole 378} \\
\hline $1-1,133-134$ & 55 & 50 & - & - & 5 & $\begin{array}{l}50 / 50 \\
54 / 46\end{array}$ & - & - & ++ & - & + & ++ & - & + & IL, $\mathrm{CH}$ \\
\hline $1-2,64-65 \mathrm{~A}$ & 46 & 42 & - & - & 4 & $\begin{array}{l}50 / 50 \\
54 / 46\end{array}$ & - & - & ++ & + & + & + & - & - & IL, CH \\
\hline $1-2,64-65$ B & 41 & 38 & - & - & 3 & $54 / 46$ & - & - & ++ & + & + & + & - & + & IL, $\mathrm{CH}$ \\
\hline $1-2,65-66$ & 43 & 39 & - & - & 4 & $54 / 46$ & - & - & ++ & + & + & ++ & - & + & $\mathrm{IL}, \mathrm{CH}$ \\
\hline $1-2,66-67$ & 33 & 30 & - & - & 3 & $\begin{array}{l}50 / 50 \\
54 / 46\end{array}$ & - & - & ++ & + & + & + & - & + & $\mathrm{IL}, \mathrm{CH}$ \\
\hline $1-2,67-68$ & 49 & 45 & - & - & 3 & $\begin{array}{l}50 / 50 \\
54 / 46\end{array}$ & - & - & ++ & + & + & + & - & + & $\mathrm{IL}, \mathrm{CH}$ \\
\hline $1-2,69-70$ & 48 & 46 & - & - & 2 & $54 / 46$ & - & - & ++ & + & + & + & - & + & IL, $\mathrm{CH}$ \\
\hline $1-2,71-72$ & 62 & 60 & - & - & 2 & $54 / 46$ & - & - & + & - & + & - & - & - & \\
\hline $3-3,122-123$ & 35 & 30 & + & - & 2 & $\begin{array}{l}50 / 50 \\
54 / 46\end{array}$ & 3 & - & ++ & - & + & + & - & + & IL, $\mathrm{CH}$ \\
\hline $6-2,18-19$ & 37 & 36 & - & - & 1 & $\begin{array}{l}50 / 50 \\
54 / 46\end{array}$ & - & - & ++ & + & + & + & - & + & IL \\
\hline $6-3,84-85$ & 48 & 42 & - & - & 6 & $\begin{array}{l}50 / 50 \\
54 / 46\end{array}$ & - & - & ++ & - & + & + & - & + & $\mathrm{IL}, \mathrm{CH}$ \\
\hline $6-3,95-96$ & 52 & 44 & - & - & 8 & $\begin{array}{l}50 / 50 \\
54 / 46\end{array}$ & - & - & ++ & + & + & + & - & - & $\mathrm{IL}, \mathrm{CH}$ \\
\hline $7-4,71-72$ & 38 & 37 & - & - & 1 & $50 / 50$ & - & - & + & + & + & + & - & + & IL \\
\hline $8-1,124-125$ & 51 & 50 & - & - & 1 & $\begin{array}{l}50 / 50 \\
54 / 46\end{array}$ & - & - & + & + & + & + & - & + & IL \\
\hline $8-2,2-3$ & 34 & 31 & - & - & 3 & $54 / 46$ & - & - & ++ & + & + & + & - & + & IL \\
\hline $8-2,13-14$ & 34 & 33 & - & - & 1 & $\begin{array}{l}50 / 50 \\
54 / 46\end{array}$ & - & - & ++ & + & + & + & - & + & IL \\
\hline $8-2,31-35$ & 33 & N.D. $\rightarrow$ & - & - & - & - & - & - & - & - & - & - & - & - & - \\
\hline $8-2,45-46$ & 27 & 27 & - & - & + & - & - & - & ++ & + & + & - & - & - & $\mathrm{IL}, \mathrm{CH}$ \\
\hline $8-2,47-48$ & 35 & N.D. $\rightarrow$ & - & - & $\overline{-}$ & - & - & - & - & - & - & - & - & - & - \\
\hline $8-2,59-60$ & 24 & 21 & - & - & 3 & $54 / 46$ & - & - & ++ & - & ++ & + & - & + & IL \\
\hline
\end{tabular}


TABLE 1 - Continued

\begin{tabular}{|c|c|c|c|c|c|c|c|c|c|c|c|c|c|c|c|}
\hline $\begin{array}{c}\text { Sample } \\
\text { (Interval in } \mathrm{cm} \text { ) }\end{array}$ & $\begin{array}{l}\text { Tot. } \\
\text { Carb. }\end{array}$ & $\begin{array}{l}\text { Calc. } \\
(\%)\end{array}$ & $\begin{array}{l}\mathrm{MgC} . \\
(\%)\end{array}$ & $\begin{array}{l}\mathrm{Mol}-\% \\
\mathrm{MgCO}_{3}\end{array}$ & $\begin{array}{l}\text { Dol. } \\
(\%)\end{array}$ & $\begin{array}{l}\mathrm{Mol}-\% \\
\mathrm{Ca} / \mathrm{Mg}\end{array}$ & $\begin{array}{l}\text { Arag. } \\
(\%)\end{array}$ & $\begin{array}{c}\text { Magn. } \\
(\%)\end{array}$ & Quar. & K-Fel. & Plag. & Gyps. & Anhy. & Pyr. & Others \\
\hline $8-2,70-71$ & 43 & N.D. $\rightarrow$ & - & - & - & - & - & - & - & - & - & - & - & - & \\
\hline $11-1,148-149$ & 41 & 37 & - & - & 4 & $\begin{array}{l}50 / 50 \\
54 / 46\end{array}$ & - & - & ++ & + & + & + & - & + & $\mathrm{IL}, \mathrm{CH}$ \\
\hline $11-2,1-2$ & 38 & 37 & - & - & 1 & $50 / 50$ & - & - & t+ & + & + & + & - & - & $\mathrm{IL}, \mathrm{CH}$ \\
\hline $11-2,28-29$ & 40 & 36 & - & - & 4 & $\begin{array}{l}50 / 50 \\
54 / 46\end{array}$ & - & - & ++ & - & + & + & - & + & IL, $\mathrm{CH}$ \\
\hline $11-2,73-74$ & 54 & 52 & - & - & 2 & $\begin{array}{l}50 / 50 \\
54 / 46\end{array}$ & - & - & ++ & - & + & - & - & - & IL, CH \\
\hline $11-2,98-99$ & 42 & 40 & - & - & 2 & $\begin{array}{l}50 / 50 \\
54 / 46\end{array}$ & - & - & ++ & - & ++ & + & - & + & IL \\
\hline $11-2,136-137$ & 45 & 44 & - & - & 1 & $\begin{array}{l}50 / 50 \\
54 / 46\end{array}$ & - & - & ++ & + & + & + & - & + & $\mathrm{IL}, \mathrm{CH}$ \\
\hline $11-4,9-10$ & 24 & 23 & - & - & 1 & $\begin{array}{l}50 / 50 \\
54 / 46\end{array}$ & - & - & ++ & + & + & + & - & + & IL, $\mathrm{CH}$ \\
\hline $11-4,22-23$ & 34 & 32 & - & - & 2 & $\begin{array}{l}50 / 50 \\
54 / 46\end{array}$ & - & - & ++ & - & + & + & - & + & $\mathrm{IL}, \mathrm{CH}$ \\
\hline $11-4,37-38$ & 31 & 29 & - & - & 2 & $54 / 46$ & - & - & t+ & + & + & - & - & + & IL \\
\hline $11-4,48-49$ & 27 & 25 & - & - & 2 & $\begin{array}{l}50 / 50 \\
54 / 46\end{array}$ & - & - & ++ & - & + & - & - & + & $\mathrm{IL}, \mathrm{CH}$ \\
\hline $11-4,64-65$ & 33 & 33 & - & - & - & - & - & - & ++ & - & + & + & - & + & $\mathrm{IL}, \mathrm{CH}$ \\
\hline $11-4,130-131$ & 30 & 21 & - & - & 9 & $\begin{array}{l}50 / 50 \\
54 / 46\end{array}$ & - & - & ++ & - & ++ & - & - & + & IL \\
\hline \multicolumn{16}{|l|}{ Hole 378A } \\
\hline $1-2,20-21$ & 34 & 27 & - & - & 7 & $\begin{array}{l}50 / 50 \\
54 / 46\end{array}$ & - & - & ++ & + & + & + & - & + & IL \\
\hline $1-3,14-15$ & 43 & 43 & - & - & - & - & - & - & ++ & + & + & - & - & - & IL \\
\hline $1-3,143-145$ & 49 & 44 & - & - & 5 & $54 / 46$ & - & - & + & + & + & - & - & - & - \\
\hline
\end{tabular}

List of abbreviations used in the table:

$\begin{array}{ll}\text { Tot. Carb. } & =\text { total carbonate } \\ \text { Calc. } & =\text { calcite } \\ \text { MgC. } & =\text { Mg-calcite } \\ \text { Dol. } & =\text { dolomite } \\ \text { Arag. } & =\text { aragonite } \\ \text { Magn. } & =\text { magnesite } \\ \text { Quar. } & =\text { quartz } \\ \text { N.D. } & =\text { not determined } \\ \text { J } & \end{array}$

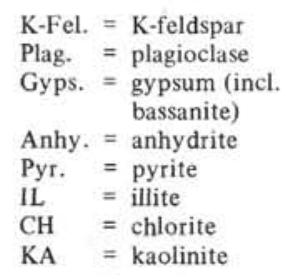

K-feldspar

Gyps. = gypsum $($ incl

Anhy. = anhydrite

Pyr. = pyrite

$\mathrm{CH}=$ chlorite

$\mathrm{KA}=$ kaolinite

$$
\begin{aligned}
\mathrm{A}= & \text { attapulgite } \\
\mathrm{HO}= & \text { hornblende } \\
\mathrm{SE}= & \text { serpentine } \\
\mathrm{HA}= & \text { halite } \\
\mathrm{BI}= & \text { bischoffite } \\
\mathrm{L}= & \text { lueneburgite } \\
\mathrm{U}= & \text { unidentified } \\
& \text { (MgSO } \left._{4 ?}\right)
\end{aligned}
$$

